IEEE/ASME Trans. Mechatronics, vol. 8, no. 4, pp. 511-513, Nov./Dec. 2003

\title{
Robust Control of Robot Manipulator by Model Based Disturbance Attenuation
}

Keywords : Robot manipulators, MBDA, position control, Liapunov function, stability.

Chong-Ho Choi and Nojun Kwak chchoi@csl.snu.ac.kr, nojunk@ieee.org Both are the members of the IEEE.

Phone: (+82-2)880-7310, Fax: (+82-2) 885-4459

School of Electrical Engineering and Computer Science, Seoul National University

San 56-1, Shinlim-dong, Kwanak-ku, Seoul 151-742 KOREA

Chong-Ho Choi is with the School of Electrical Engineering and Computer Science and the Automation and Systems Research Institute, Seoul National Univ., Seoul, Korea.

Nojun Kwak is a Ph.D student in the School of Electrical Engineering and Computer Science, Seoul National Univ., Seoul,Korea.

The corresponding author is Chong-Ho Choi and his e-mail address is underlined.

This project is partially supported by the Brain Science and Engineering Program of the Korea Ministry of Science and Technology and the Brain Korea 21 Program of the Korea Ministry of Education. 


\begin{abstract}
In this letter, a model based disturbance attenuator (MBDA) for robot manipulators is proposed and the stability of the MBDA in robot positioning problems is proved via Liapunov's direct method. This method does not require an accurate model of a robot manipulator and takes care of disturbances or modelling errors so that the plant output remains relatively unaffected by them. The output error due to the gravity or constant disturbance can be effectively eliminated by this method.
\end{abstract}

\title{
Keywords
}

Robot manipulators, MBDA, position control, Liapunov function, stability.

\section{INTRODUCTION}

To achieve high performance in controlling robots, much research has been conducted under the assumption that the dynamics of robot systems are exactly known. But this assumption is not usually satisfied because it is very difficult to obtain an exact robot model due to its nonlinear dynamic structure and modelling uncertainties. To overcome these problems, a model based disturbance attenuator for robot manipulators is proposed and its asymptotic stability is proved in the following. It is a generalization of [1] for robot manipulators and a preliminary result without the stability analysis was presented in [2].

\section{The MBDA Controller And Its Stability}

In the absence of friction and disturbances, the dynamics of an $n$ degree of freedom robot manipulator is given by the Lagrange-Euler vector equation:

$$
M(q) \ddot{q}+C(q, \dot{q}) \dot{q}+g(q)=\tau
$$

where $q, \dot{q}, \ddot{q} \in \Re^{n}$ are the vectors of generalized position, velocity, and acceleration respectively, of $n$ links, $M(q) \in \Re^{n \times n}$ is the inertia matrix which is positive definite and symmetric, $C(q, \dot{q}) \in \Re^{n \times n}$ accounts for the centrifugal and Coriolis terms, $g(q) \in \Re^{n}$ is the gravity term, and $\tau \in \Re^{n}$ is the generalized torque acting on the links.

The equation of motion (1) has the following properties [3].

Property 1: The matrix $N(q, \dot{q})=\dot{M(q)}-2 C(q, \dot{q})$ is skew symmetric. Thus,

$$
\dot{M(q)}=C(q, \dot{q})+C^{T}(q, \dot{q})
$$


Property 2: There exists a positive constants $k_{c}$ such that

$$
\|C(q, \dot{q})\| \leq k_{c}\|\dot{q}\|, \quad \forall q, \dot{q} \in \Re^{n}
$$

Property 3 : There exists a positive constant $k_{g}$ satisfying

$$
\left\|\frac{\partial g(q)}{\partial q}\right\|<k_{g}, \quad \forall q \in \Re^{n} \quad \text { and } \quad\|g(x)-g(y)\| \leq k_{g}\|x-y\|, \quad \forall x, y \in \Re^{n} .
$$

Figure 1 shows the structure of MBDA, where $\mathrm{P}$ is a plant, $\mathrm{M}$ is a model for the plant P. The vectors $q, q_{0}, q_{d} \in \Re^{n}$ are a position vector of the plant, a position vector of the model, and a desired position vector respectively, $\tau, \tau_{0} \in \Re^{n}$ are input torques for plant and model. In the figure, $\mathrm{K}, \mathrm{K}_{1}$, and $\mathrm{K}_{2}$ are feedback gain matrices of appropriate dimensions. The conventional PD gains are used for $K$ and $K_{1}$, and only $D$ gains are used for $K_{2}$. If $\mathrm{K}_{2}=0$, it is the same structure as in [1].

Let $\boldsymbol{q} \triangleq\left[q^{T}, \quad\left(q_{0}-K_{p 1}^{-1} g\left(q_{d}\right)\right)^{T}\right]^{T}$, and $T \triangleq\left[\left(\tau-g\left(q_{d}\right)\right)^{T}, \quad \tau_{0}^{T}\right]^{T}$. Then the robot dynamics of the overall system is

$$
\begin{aligned}
T & =\left[\begin{array}{cc}
M(q) & 0 \\
0 & M_{0}\left(q_{0}\right)
\end{array}\right]\left[\begin{array}{c}
\ddot{q} \\
\ddot{q_{0}}
\end{array}\right]+\left[\begin{array}{cc}
C(q, \dot{q}) & 0 \\
0 & C_{0}\left(q_{0}, \dot{q}_{0}\right)
\end{array}\right]\left[\begin{array}{c}
\dot{q} \\
\dot{q_{0}}
\end{array}\right]+\left[\begin{array}{c}
g(q)-g\left(q_{d}\right) \\
0
\end{array}\right] \\
& =\boldsymbol{M}(\boldsymbol{q}) \ddot{\boldsymbol{q}}+\boldsymbol{C}(\boldsymbol{q}, \dot{\boldsymbol{q}}) \dot{\boldsymbol{q}}+\boldsymbol{g}\left(\boldsymbol{q}, \boldsymbol{q}_{\boldsymbol{d}}\right),
\end{aligned}
$$

where the subscript " 0 " is used to represent the terms related to the model. Note that we omit the gravity term in the model dynamics.

Assuming a constant desired position vector, i.e., $\dot{q}_{d}(t)=0$ for $t>0$, the feedback dynamics of the system in Fig. 1 becomes

$$
T=-\left[\begin{array}{cc}
K_{p}+K_{p 1} & -K_{p 1} \\
K_{p} & 0
\end{array}\right]\left[\begin{array}{c}
\tilde{q} \\
\tilde{q_{m}}
\end{array}\right]-\left[\begin{array}{cc}
K_{d}+K_{d 1} & -K_{d 1} \\
K_{d}-K_{d 2} & K_{d 2}
\end{array}\right]\left[\begin{array}{c}
\dot{\tilde{q}} \\
\dot{\tilde{q}}
\end{array}\right]=-K_{\boldsymbol{p}} \tilde{\boldsymbol{q}}-\boldsymbol{K}_{\boldsymbol{d}} \dot{\boldsymbol{q}} .
$$

Here, $\tilde{q} \triangleq q-q_{d}, \tilde{q_{0}} \triangleq q_{0}-q_{d}, \tilde{q_{m}} \triangleq \tilde{q_{0}}-K_{p 1}^{-1} g\left(q_{d}\right)$ and $\tilde{\boldsymbol{q}} \triangleq\left[\tilde{q}^{T},{\tilde{q_{m}}}^{T}\right]^{T}$. The diagonal matrices $K_{p}\left(K_{p 1}\right)$ and $K_{d}\left(K_{d 1}\right)$ are $\mathrm{P}$ and $\mathrm{D}$ gains of $\mathrm{K}\left(\mathrm{K}_{1}\right)$, and the diagonal matrix $K_{d 2}$ is D gain of $K_{2}$ in Fig. 1 . In the above equations (2) and (3), $K_{\boldsymbol{p}}, K_{\boldsymbol{d}}, \boldsymbol{M}(\boldsymbol{q}), \boldsymbol{C}(\boldsymbol{q}, \dot{\boldsymbol{q}})$, and $\boldsymbol{g}\left(\boldsymbol{q}, \boldsymbol{q}_{\boldsymbol{d}}\right)$ are appropriately defined.

From these equations, the following closed loop dynamic equation of the MBDA system in Fig. 1 is obtained:

$$
M(q) \ddot{q}+C(q, \dot{q}) \dot{q}+g\left(q, q_{d}\right)=-K_{p} \tilde{q}-K_{d} \dot{q}
$$


To carry out the stability analysis, we consider the following candidate Liapunov function:

$$
V=\frac{1}{2}\left(\tilde{\boldsymbol{q}}^{T} \boldsymbol{K} \tilde{\boldsymbol{q}}+\dot{\boldsymbol{q}}^{T} \boldsymbol{M} \dot{\boldsymbol{q}}\right)+\frac{1}{\gamma} \tilde{\boldsymbol{q}}^{T} \boldsymbol{M} \dot{\boldsymbol{q}}=\frac{1}{2} \boldsymbol{x}^{T}\left[\begin{array}{cc}
\boldsymbol{K} & \frac{1}{\gamma} \boldsymbol{M} \\
\frac{1}{\gamma} \boldsymbol{M} & \boldsymbol{M}
\end{array}\right] \boldsymbol{x} \triangleq \boldsymbol{x}^{T} \boldsymbol{L} \boldsymbol{x}
$$

where $K$ is a symmetric positive definite constant matrix and $\gamma$ is a positive constant and $\boldsymbol{x} \triangleq\left[\tilde{\boldsymbol{q}}^{T}, \dot{\boldsymbol{q}}^{T}\right]^{T}$.

Using the following inequality

$$
2\left\|u^{T} A v\right\| \leq a_{1} u^{T} A u+\frac{1}{a_{1}} v^{T} A v, \quad a_{1}>0
$$

which holds for any positive definite matrix $A \in \Re^{n \times n}$ and for any vector $u, v \in \Re^{n}, V$ can be shown to be a valid Liapunov function such that it becomes positive definite

$$
V \geq \frac{1}{2} \tilde{\boldsymbol{q}}^{T}\left(\boldsymbol{K}-\frac{a_{1}}{\gamma} \boldsymbol{M}\right) \tilde{\boldsymbol{q}}+\frac{1}{2}\left(1-\frac{1}{a_{1} \gamma}\right) \dot{\boldsymbol{q}}^{T} \boldsymbol{M} \dot{\boldsymbol{q}} \geq 0 .
$$

if for an arbitrary positive constant $a_{1}$, the following condition holds

$$
a_{1} \gamma>1, \quad K-\frac{a_{1}}{\gamma} \boldsymbol{M}>0
$$

Theorem: Let $K_{p}=K_{p 1}, \quad K_{d}=K_{d 1}+K_{d 2}$ and

$$
\boldsymbol{K}=\left[\begin{array}{cc}
2 K_{p}+\frac{1}{\gamma}\left(K_{d}+K_{d 1}\right) & \left.-K_{p}+\frac{1}{\gamma} K_{d 1}\right) \\
-K_{p}+\frac{1}{\gamma} K_{d 1} & \frac{1}{\gamma} K_{d 2}
\end{array}\right]
$$

Also let $\Omega \triangleq\{\boldsymbol{x}:\|\boldsymbol{x}\|<b \sqrt{\underline{\lambda} / \bar{\lambda}}\}$, where $\underline{\lambda}$ and $\bar{\lambda}$ are the minimum and maximum eigenvalues of $\boldsymbol{L}$ respectively.

For a constant input $q_{d}$, the system in Fig.1 is asymptotically stable at the origin $\boldsymbol{x}_{0}=\mathbf{0}$ and $\Omega$ is a region of asymptotic stability, if the following conditions are satisfied.

$$
\begin{aligned}
& K_{d}+K_{d 1}-\frac{1}{\gamma} M-\frac{k_{c} b}{\gamma} I_{n}-\frac{a_{3} k_{g}}{2} I_{n}>0 \\
& K_{d 2}-\frac{1}{\gamma} M_{0}-\frac{k_{c 0} b}{\gamma} I_{n}-a_{2} E>0 \\
& 2 K_{p}-k_{g} I_{n}-\frac{\gamma}{a_{2}} E-\frac{\gamma k_{g}}{2 a_{3}} I_{n}>0
\end{aligned}
$$

Here $a_{2}, a_{3}$ and $b$ are arbitrary positive constants, $I_{n}$ is an $n \times n$ identity matrix, and

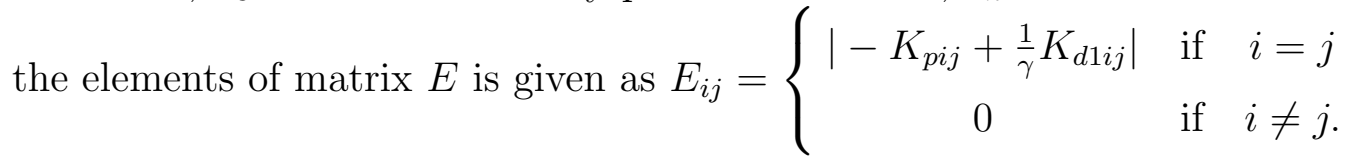


The constant $k_{c 0}$ is chosen to meet the Property 2 for model dynamics such that $\left\|C_{0}\left(q_{0}, \dot{q}_{0}\right)\right\| \leq k_{c 0}\left\|\dot{q}_{0}\right\|$.

Proof: Differentiating (5), $\dot{V}$ becomes

$$
\begin{aligned}
\dot{V}= & \frac{1}{2}\left(2 \dot{\boldsymbol{q}}^{T}(\boldsymbol{M} \ddot{\boldsymbol{q}}+\boldsymbol{K} \tilde{\boldsymbol{q}})+\dot{\boldsymbol{q}}^{T} \dot{\boldsymbol{M}} \dot{\boldsymbol{q}}\right)+\frac{1}{\gamma}\left(\dot{\boldsymbol{q}}^{T} \boldsymbol{M} \dot{\boldsymbol{q}}+\tilde{\boldsymbol{q}}^{T} \dot{\boldsymbol{M}} \dot{\boldsymbol{q}}+\tilde{\boldsymbol{q}}^{T} \boldsymbol{M} \ddot{\boldsymbol{q}}\right) \\
= & \dot{\boldsymbol{q}}^{T}\left(\boldsymbol{K}-\boldsymbol{K}_{\boldsymbol{p}}-\frac{1}{\gamma} \boldsymbol{K}_{\boldsymbol{d}}^{T}\right) \tilde{\boldsymbol{q}}-\dot{\boldsymbol{q}}^{T}\left(\boldsymbol{K}_{\boldsymbol{d}}-\frac{1}{\gamma} \boldsymbol{M}\right) \dot{\boldsymbol{q}}-\dot{\boldsymbol{q}}^{T} \boldsymbol{g}\left(\boldsymbol{q}, \boldsymbol{q}_{\boldsymbol{d}}\right)-\frac{1}{\gamma}\left(\tilde{\boldsymbol{q}}^{T} \boldsymbol{K}_{\boldsymbol{p}} \tilde{\boldsymbol{q}}-\tilde{\boldsymbol{q}}^{T} \boldsymbol{C}^{T} \dot{\boldsymbol{q}}+\tilde{\boldsymbol{q}}^{T} \boldsymbol{g}\left(\boldsymbol{q}, \boldsymbol{q}_{\boldsymbol{d}}\right)\right) \\
= & 2 \dot{q}_{0}^{T}\left(-K_{p}+\frac{1}{\gamma} K_{d 1}\right) \tilde{q}-\dot{q}^{T}\left(K_{d}+K_{d 1}-\frac{1}{\gamma} M\right) \dot{q}+\frac{1}{\gamma} \tilde{q}^{T} C^{T} \dot{q}-\dot{q}_{0}^{T}\left(K_{d 2}-\frac{1}{\gamma} M_{0}\right) \dot{q}_{0} \\
& +\frac{1}{\gamma} \tilde{q}_{m}^{T} C_{0}^{T} \dot{q}_{0}-\frac{2}{\gamma} \tilde{q}^{T} K_{p} \tilde{q}-\dot{q}^{T}\left\{g(q)-g\left(q_{d}\right)\right\}-\frac{1}{\gamma} \tilde{q}^{T}\left\{g(q)-g\left(q_{d}\right)\right\} .
\end{aligned}
$$

The second equality is from Properties 1 and (4) and the third equality is obtained using $K_{p}=K_{p 1}, K_{d}=K_{d 1}+K_{d 2}$ and the definition of $\boldsymbol{K}$. Using Property 2 and 3, the terms related to the Corioris and gravity forces are bounded such that

$$
\begin{aligned}
& \left\|\tilde{q}^{T} C^{T} \dot{q}\right\| \leq k_{c}\|\dot{q}\|^{2}\|\tilde{q}\|, \quad\left\|\tilde{q_{m}} C_{0}^{T} \dot{q_{0}}\right\| \leq k_{c 0}\left\|\dot{q_{0}}\right\|^{2}\left\|\tilde{q_{m}}\right\| \\
& \left\|\dot{q}^{T}\left(g(q)-g\left(q_{d}\right)\right)\right\|<k_{g}\|\dot{q}\|\left\|q-q_{d}\right\|=k_{g}\|\dot{q}\|\|\tilde{q}\| \\
& \left\|\tilde{q}^{T}\left(g(q)-g\left(q_{d}\right)\right)\right\|<k_{g}\|\tilde{q}\|\left\|q-q_{d}\right\|=k_{g}\|\tilde{q}\|^{2}
\end{aligned}
$$

and this leads to

$$
\begin{aligned}
\dot{V} \leq & 2 \dot{q}_{0}^{T}\left(-K_{p}+\frac{1}{\gamma} K_{d 1}\right) \tilde{q}-\dot{q}^{T}\left(K_{d}+K_{d 1}-\frac{1}{\gamma} M-\frac{k_{c}\|\tilde{q}\|}{\gamma} I_{n}\right) \dot{q} \\
& -\dot{q}_{0}^{T}\left(K_{d 2}-\frac{1}{\gamma} M_{0}-\frac{k_{c 0}\left\|\tilde{q_{m}}\right\|}{\gamma} I_{n}\right) \dot{q}_{0}-\frac{2}{\gamma} \tilde{q}^{T} K_{p} \tilde{q}+k_{g}\|\dot{q}\|\|\tilde{q}\|+\frac{1}{\gamma} k_{g}\|\tilde{q}\|^{2} \\
\leq & -\dot{q}^{T}\left(K_{d}+K_{d 1}-\frac{1}{\gamma} M-\frac{k_{c}\|\tilde{q}\|}{\gamma} I_{n}-\frac{a_{3} k_{g}}{2} I_{n}\right) \dot{q}-\dot{q}_{0}^{T}\left(K_{d 2}-\frac{1}{\gamma} M_{0}-\frac{k_{c 0}\left\|\tilde{q_{m}}\right\|}{\gamma} I_{n}-a_{2} E\right) \dot{q}_{0} \\
& -\frac{1}{\gamma} \tilde{q}^{T}\left(2 K_{p}-k_{g} I_{n}-\frac{\gamma}{a_{2}} E-\frac{\gamma k_{g}}{2 a_{3}} I_{n}\right) \tilde{q} .
\end{aligned}
$$

The second inequality is by applying (6) to every non-quadratic terms. Note that if condition (8) holds, $\dot{V}$ becomes negative semi-definite for all the points within $\Gamma=\{x$ : $\|x\|<b\}$.

Because $\underline{\lambda}\|\boldsymbol{x}\|^{2} \leq V \leq \bar{\lambda}\|\boldsymbol{x}\|^{2}$, the path of $\boldsymbol{x}$ starting on $\Omega$ will not leave the region $\Gamma$. The necessary condition for $\dot{V}=0$ is $\boldsymbol{x}^{T}=\left[\tilde{q}, \tilde{q}_{m}, \dot{q}, \dot{q}_{0}\right]=\left[0, \tilde{q}_{m}, 0,0\right]$. For this point to be stable, $\tilde{q}_{m}$ must be zero, because from (4) it becomes $M \ddot{q}+C \dot{q}+g(q)=-\left(K_{p}+K_{p 1}\right) \tilde{q}-$ $\left(K_{d}+K_{d 1}\right) \dot{q}+K_{p 1} \tilde{q}_{0}+K_{d 1} \dot{q}_{0}$. Thus the origin $\boldsymbol{x}_{0}=\mathbf{0}$ is the only point contained in the 
largest invariant set in $R=\{x: x \in \Omega, \dot{V}=0\}$. Finally, using the Theorem VI of [4], the origin is asymptotically stable and every point in $\Omega$ tends to the origin as $t \rightarrow \infty$.

Although the conditions in (8) seem hard to be satisfied, these conditions can be easily met if $K_{p}, K_{d 1}$, and $K_{d 2}$ can be set sufficiently large. Note that at the equilibrium point, the position error of the plant is $\tilde{q}=0$, but the position error of the model becomes $\tilde{q}_{0}=$ $K_{p 1}^{-1} g\left(q_{d}\right)$. The gravitational force $g(q)$, which is a disturbance, is completely compensated by the position error of the model and do not affect the plant output in the steady state, because the model feedback gain $\mathrm{K}_{2}$ does not contain a proportional component.

In many robot manipulators, not only the Coriolis/centrifugal forces but also the inertia matrix is not easy to estimate. For this case, we can model a robot with a constant inertia matrix $M_{0}$ using only its diagonal components and set $C_{0}=0$. This simplifies the modelling process greatly without degrading the performance. A simulation result for a two-link robot with this method was presented in [2].

\section{Conclusions}

In this letter, a new method for controlling robot manipulators is proposed and its stability is proved. The proposed method is easy to implement and very robust in regard to modelling errors and disturbances. It consists of a model in parallel with the plant. In the presence of disturbances, this method attenuates the disturbance significantly. This MBDA controller has both the advantage of a PD controller in that it is asymptotically stable and the advantage of a PID controller which can eliminate steady state errors due to modelling errors or disturbances.

\section{REFERENCES}

[1] B.-K. Choi, C.-H. Choi and H. Lim, "Model based disturbance attenuation for CNC machining centers," IEEE/ASME Trans. Mechatronics, vol. 4, no. 2, pp. 157-168, June 1999.

[2] C.-H Choi and N. Kwak, "Disturbance attenuation in robot control," Proc. of Int. Conf. on Robotics and Automation, pp.2560-2565, Seoul, May 2001.

[3] F. L. Lewis, C. T. Abdallah, and D. M. Dawson, Control of Robot Manipulators, NY, Macmillan, 1993.

[4] J. La Salle and S. Lefschetz Stability by Liapunov's Direct Method with Applications, Academic Press, N,Y., 1961. 


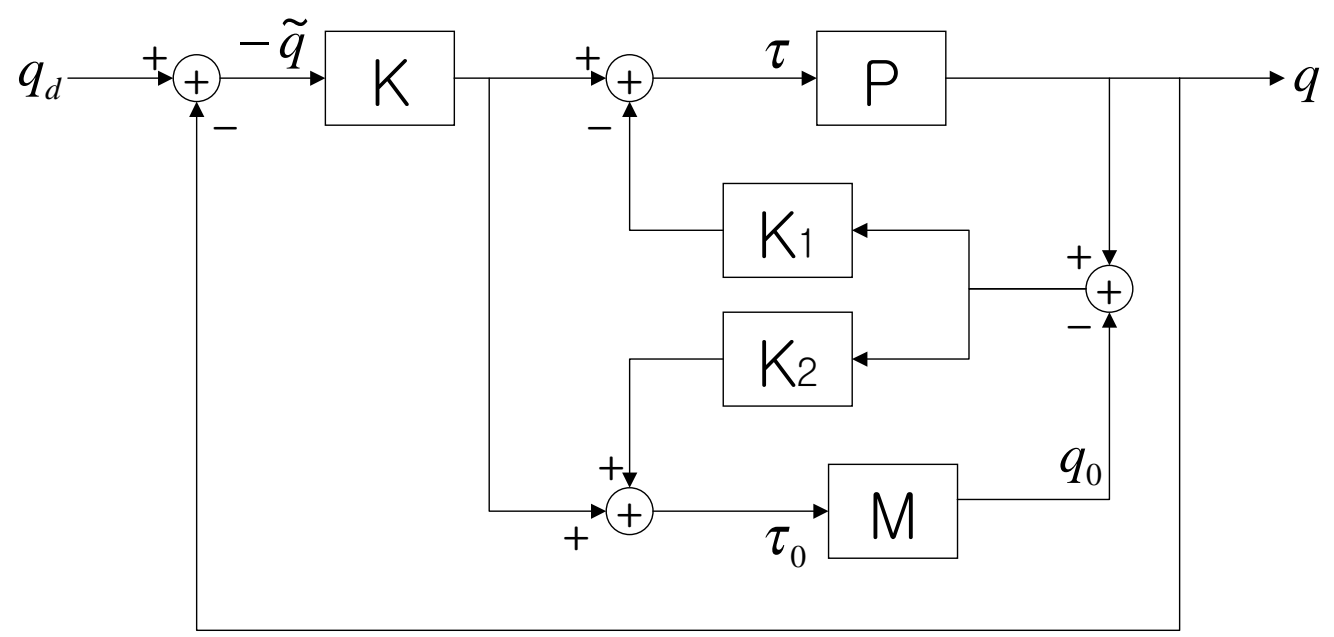

Fig. 1. MBDA Controller 